\title{
Axel Mobility Platform for Steep Terrain Excursions and Sampling on Planetary Surfaces
}

\author{
Issa A.D. Nesnas \\ Jet Propulsion Laboratory \\ Californi a Institute of T echnology \\ Pasadena, California 91109 \\ 818-354-9709 \\ nesnas@jplnasagov
}

\author{
Pablo Abad-Manterola, Jeffrey A. Edlund \\ Joel W. Burdick \\ California Institute of Technology \\ Pasadena, CA 91125 \\ pablo@caltech.edu \\ jedlund@caltech.edu \\ jwb@robotics.caltech.edu
}

Abstract-The recent discovery of bright new deposits in two crater gullies on Mars suggests that water still flows in brief spurts on Mars. In this paper, we will present the Axel rover (Figure 1) that was developed to access and sample such deposits on the inside of steep crater walls. Axel is a tethered rover that can be a payload on a lander or a larger rover. The primary features of Axel are its minimal complexity and robustness to the treacherous terrain of sites that are of scientific interest. Using a symmetrical design with three actuators, Axel is capable of operating upside down and right side up, enabling it to descend over crater promontories. With its actuated trailing link, Axel can operate on both flat and sloped terrains. Using a sampling device mounted on the trailing link, Axel can collect and store terrain samples and return to its host platform for detailed scientific sample analysis. We will present our preliminary results that demonstrated Axel's ability to traverse both flat and sloped rocky terrain including $90^{\circ}$ vertical cliffs and collecting soil samples on slopes ranging from $10^{\circ}-40^{\circ}$ in the JPL Mars Yard. ${ }^{12}$

\section{TABLE OF CONTENTS}

1. Motivation 1

2. BACKGR OUND 3

3. THE TETHFRED AXEL CONCEPT .......................4

4. THEORETICAL ANALYSIS ................................6

5. THE AXFL Platform PRototyPe ................ 7

6. EXPERIMENTAL RESUL TS.................................

7. FUTURE WORK.................................................

8. SUMMARY.........................................................

9. ACKNOWLEDGMENTS ...................................10

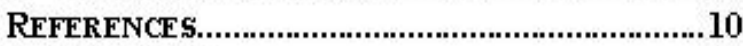

BIOGRAPHIES.......................................................

\section{Mot IVAT ION}

Recently formed bright deposits were discovered by comparing images taken from the Mars Global Surveyor orbiting spacecraft over several years. This discovery suggests that water may still flow in brief spurts on Mars and has sparked interest in developing robotic platforms that can access and sample such deposits.

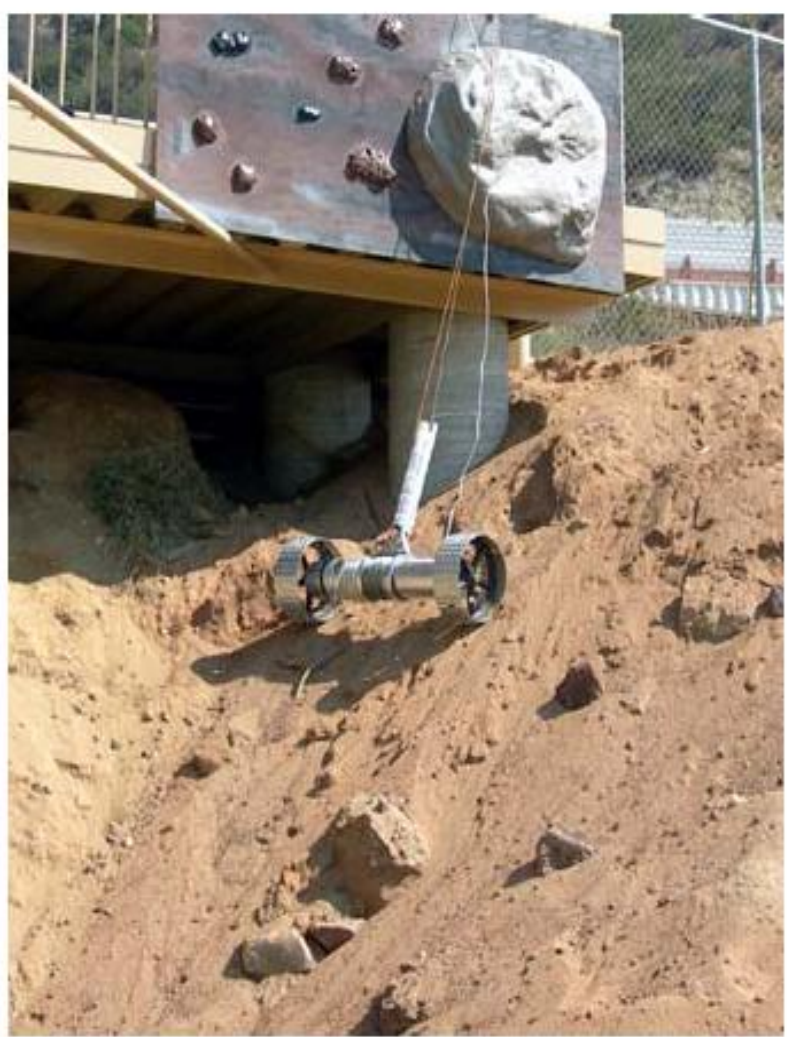

Figure $1-$ Axel driving down a $40^{\circ}$ rocky slope in the JPL Mars Yard

The first deposits shown in Figure 2 appeared on the inside wall of an unnamed crater in Terra Sirenum at 36.6 degrees south, 161.8 degrees west. These deposits are several hundred meters down from the rim of the crater, where the terrain has a slope of approximately $25^{\circ}$.

Figure 3 shows the second gully deposit that was found in an unnamed crater in the Centauri Montes region, which is located near 38.7 degrees south latitude and 263.3 degrees west longitude. Similar to the first discovery, these deposits appeared on the inside crater wall several hundred meters down from the crater rim and on a sloped terrain.

\footnotetext{
' 1-42441488-1/08\$25.00 92008 IEEE.

${ }^{2}$ IEEEAC paper \#1216, Version 6, Updated October 27, 2007
} 

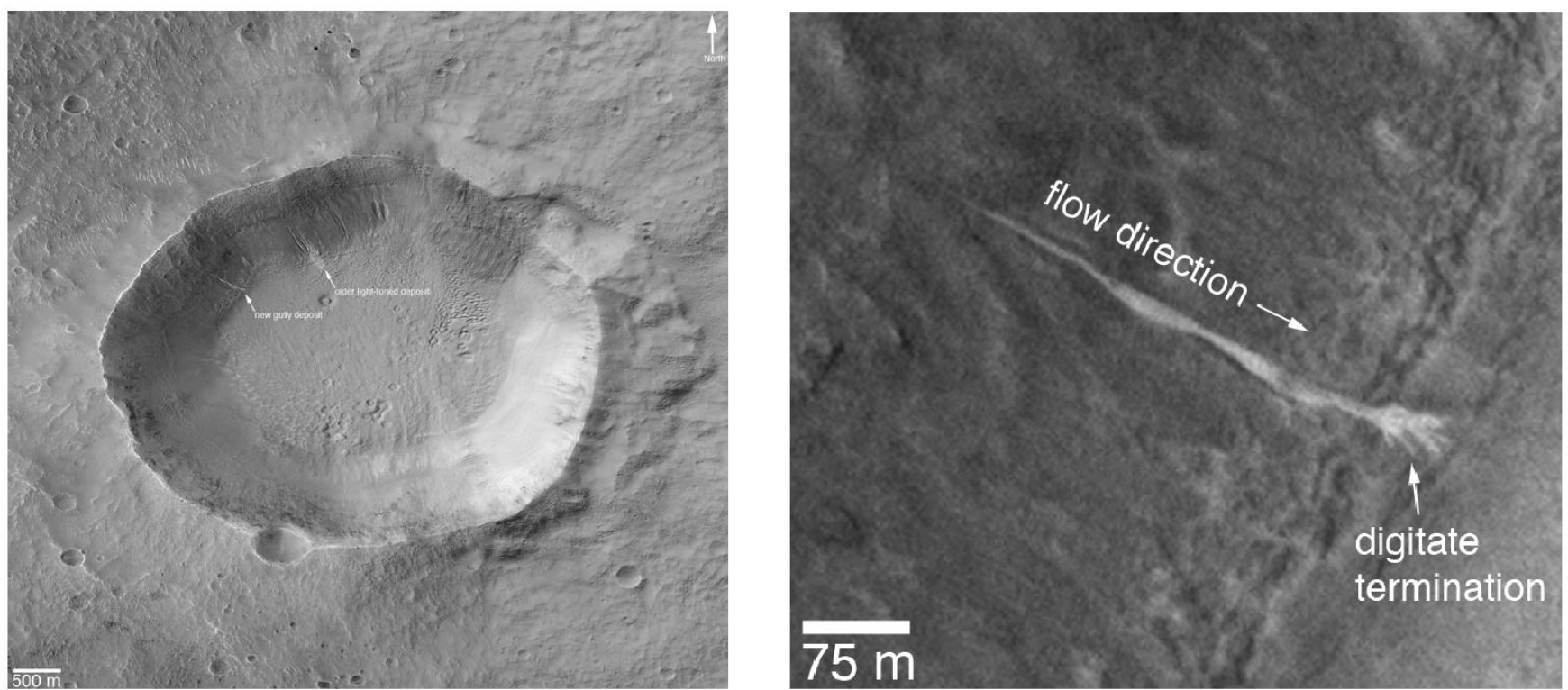

Figure 2-The unnamed crater in Terra Sirenum where the first gully deposit was found (left), an enlarged image showing details of the new, light-toned gully deposit reaching the crater floor (right). The images were acquired by the Mars Global Surveyor camera between 2005 and 2006.

To be able to access and cache samples from such deposits, we developed the Axel rover to descend down the crater's steep interior walls. We designed the rover with a capability to descend over vertical crater promontories such as those found at Cape St. Vincent in Figure 4, which were imaged by the Opportunity rover at Victoria crater on Mars. The material at the top of the promontory consists of loose, jumbled rock, which abruptly transitions to bedrock, the top of which is marked by a bright band of rock visible around the entire crater. Scientists have expressed interest in closer analyses of such layers of bedrock. We designed Axel to be able to access and closely examine such layers using its onboard sensors.
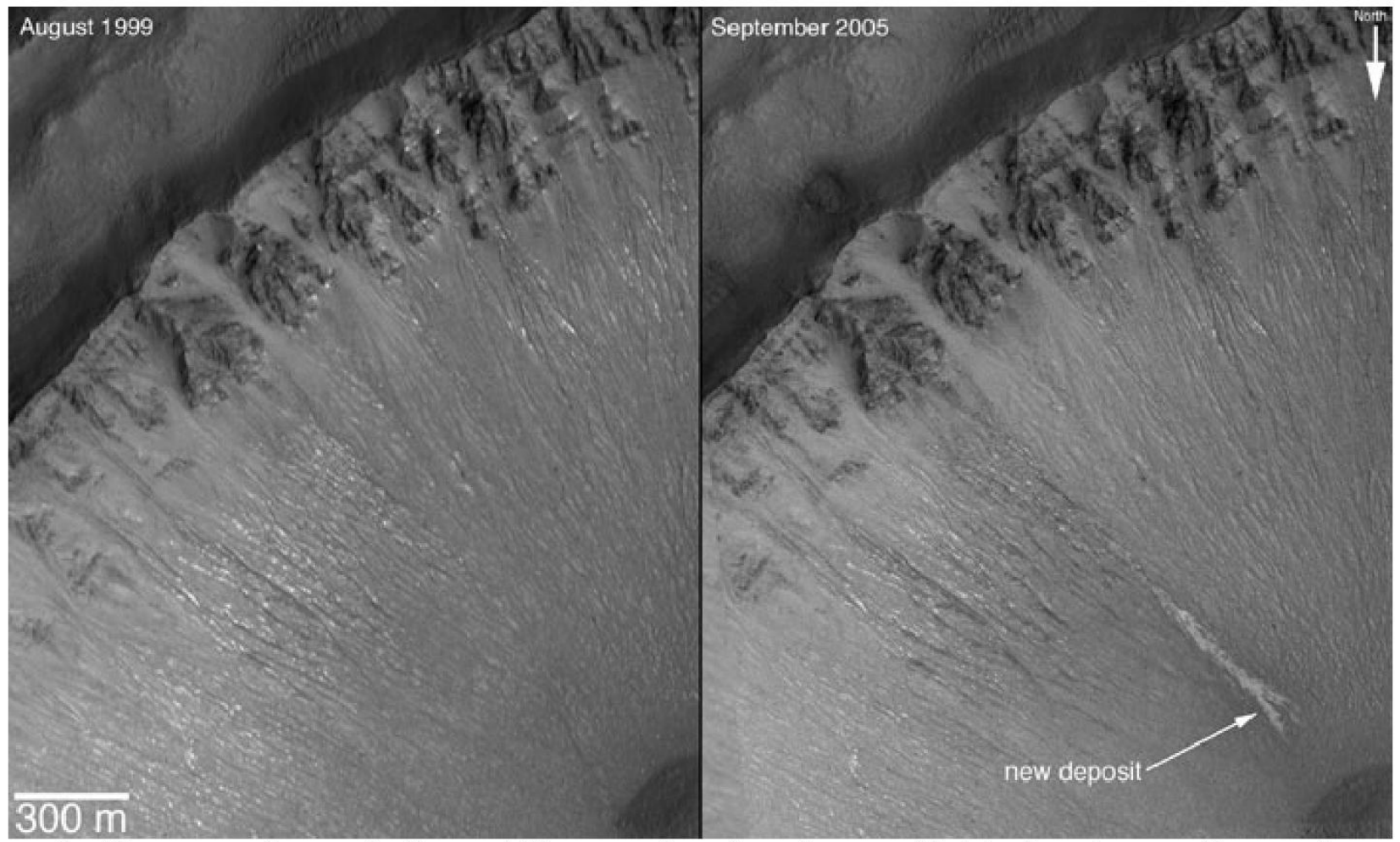

Figure 3-The unnamed crater in Centauri Montes region where the second bright deposits were discovered. 


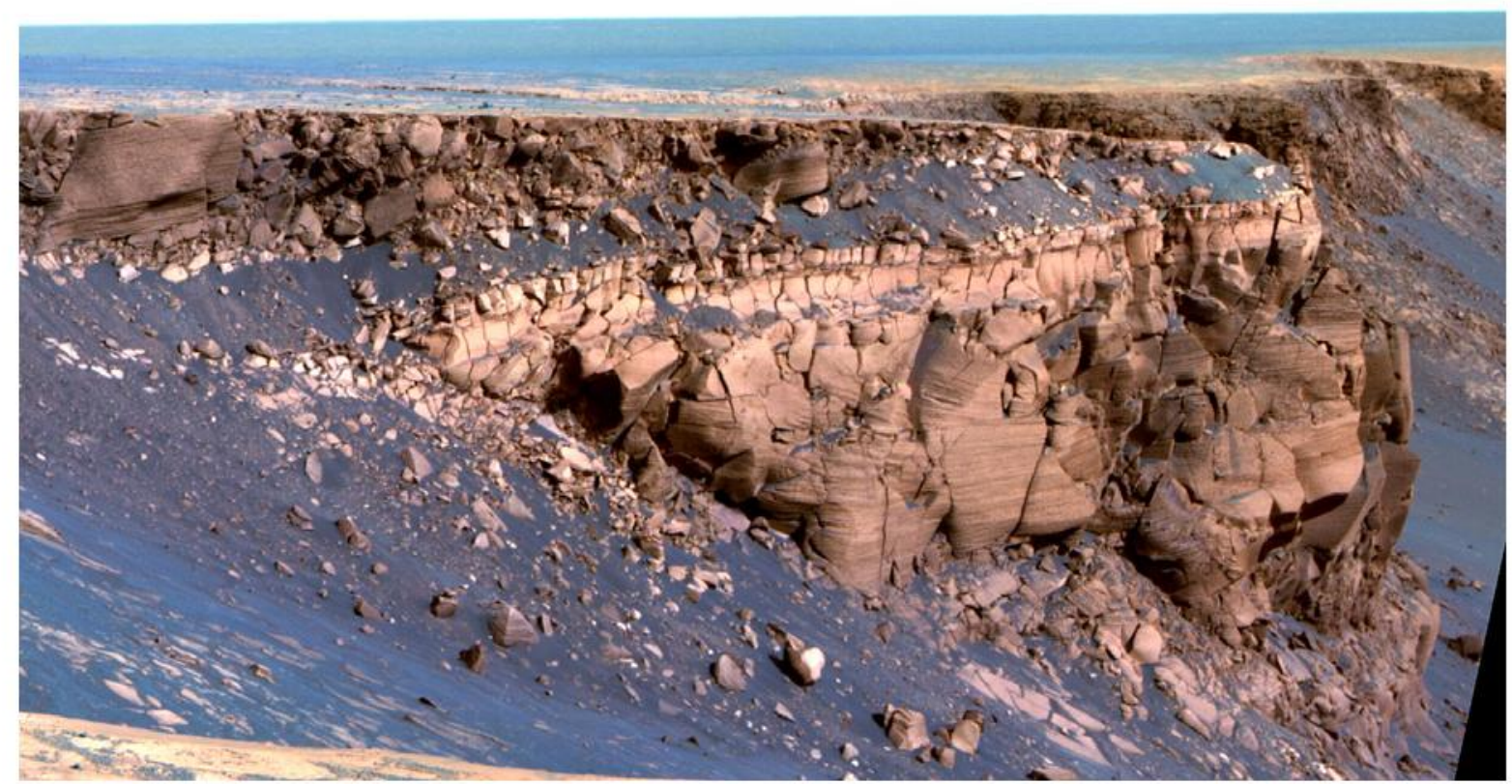

Figure 4-Cape St. Vincent is one of the many promontories that jut out from the walls of Victoria Crater, Mars (false colors used to visually separate different layers).

Transitioning from an overhang to a sloped or flat terrain presents a major challenge for robots because one cannot predict which way they will land. This requires the robot to operate from an inverted position. To meet this requirement, we designed Axel to be symmetrical, thus giving it the ability to operate upside down and right side up without added complexity.

Despite the fact that the recently discovered bright deposits are closer to the crater floor, accessing them from the top of the crater has several advantages. First, it allows for the use of a tether to aid in traversing the steep terrain. Ascending from the bottom is more difficult and would require firm soil to prevent the rover from loosing its grip and falling. It is hard to know the soil firmness a priori especially at crater floors. Second, descending from the top places fewer requirements on the precision landing of a payload and offers a larger landscape for the host platform to explore. Third, it provides easier access to vertical drops that are often closer to the rim of the crater as shown in Figure 4. Therefore, the preferred option rover for reliable and safe operations was to access these sites from the top using a small rover tethered to a host lander or larger.

In Section 2, we will describe the history of the Axel concept and other related efforts. In Section 3 , we will provide a detailed description of the tethered Axel concept. In Section 4, we will describe the first Axel prototype and in the following section, we will capture the results of the preliminary experiments that we carried out in the JPL Mars Yard. We will conclude with a section on future work.

\section{BACKGROUND}

The concept of a minimally actuated rover for planetary exploration emerged as far back as the $1970 \mathrm{~s}$. The Axel rover system was independently conceived in October 1999 [3] in response to a NASA research announcement seeking to increase the robustness of robots through modular and reconfigurable systems. The initial idea of Axel was to separate the mobility elements from the payload elements, since the mobility elements are more likely to fail. The separation of the two elements makes the overall rover system modular and reconfigurable. As a result, we developed the concept of a minimalist rover that can dock to and carry stationary payloads. We will cover this concept in more detail in Section 7 .

Researchers at the University of Minnesota independently developed a two-wheeled robot, called Scout, which was first published in April 2000 [2]. Each scout is a small cylindrical explorer (a few centimeters in diameter) that is ejected from a cannon mounted on a traditional rover platform. These tiny rovers are controlled by embedded microprocessors and sensors. In addition to exploring surrounding areas (mainly flat terrain), these cylindrical explorers can hop a few centimeters over small barriers. Due to their minimalist designs, Scout and Axel share several mobility and sensing features. Among the differences is the $360^{\circ}$ actuated link that enables Axel to control its body pitch. A commercially available robot from Probotics, Inc. [4] also used the concept of two-wheeled locomotion. 


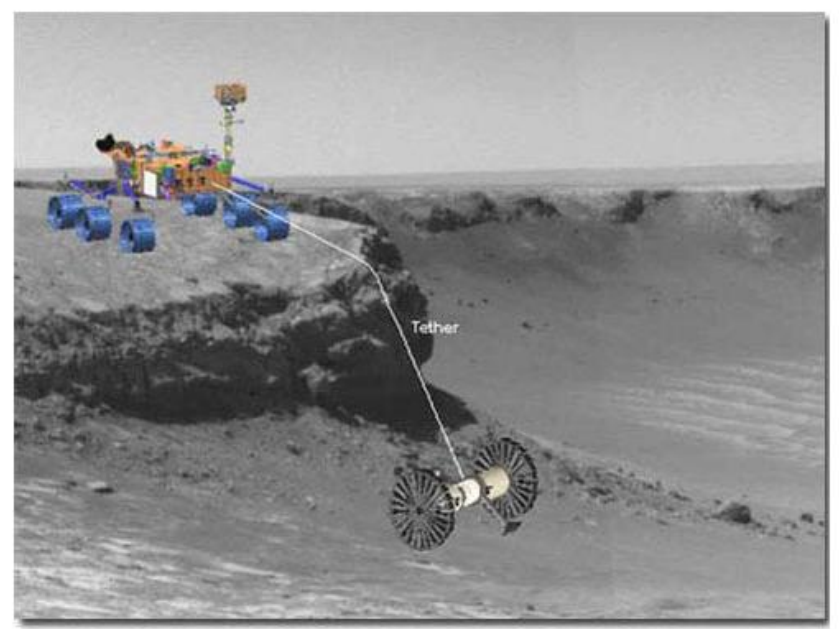

Figure 5-An overall mission concept with Axel tethered to a Marsupial rover. The rover pictures are not to scale.

Another steep terrain robot is the STAR four-legged climber [5], which uses ultrasonic drills to create foot-holds in solid rock and other materials. STAR is specialized for climbing. This type of rover could potentially cling to the bottom of an overhanging rock. However, with looser materials on slopes, the rover would be unable to hold its weight. With its legged configuration, STAR is a complex robot with high power requirements.

Tethered rovers have previously been used to explore the interior of craters. The most notable example is the Dante II rover [1] that descended into the Mt. Spurr crater. Dante II is a four legged frame walker robot. It carried a $300 \mathrm{~m}$ tether with an onboard winch, which always maintained tether tension. Care was taken to ensure proper unwinding and winding of the tether. Its large form $(3.7 \mathrm{~m} \times 2.3 \mathrm{~m} \times$ $3.7 \mathrm{~m}, 770 \mathrm{~kg}$ robot; $130 \mathrm{~kg}$ payload) allowed it to carry seven video cameras, a scanning laser rangefinder, gas detection sensors, and thermocouples. It could step onto a $1.3 \mathrm{~m}$ high boulder from flat ground and was able to rappel down any slope that did not leave it free hanging $(<=90)$. However, it could only handle a $30^{\circ}$ cross slope. Its mission ultimately came to an end when it fell over on its side and was unable to right itself. Such an experience underscores the need to be able to operate such rovers in any stable state.

Another tethered rover concept is the Cliff-bot system [6] that uses a total of four wheeled rovers and two tethers to allow one of the rovers to traverse a slope of $70^{\circ}$ or less. Two of the rovers act as "Anchor-bots" and contain winches to control the tethers. The two tethers allow the descending rover ("Cliff-bot") to move back and forth along the cliff face as well as ascend and descend. However, unreeling from the top of the cliff causes tether abrasion. The fourth robot ("Recon-bot") observes the progress from the top of the cliff and reports perceived obstacles.
Using a single tether has some advantages over multiple ones. Not only does it halve the mass of the tether and winch, it also reduces the risk of tether entanglement and the overall complexity of tether management. Given the constraints on flight missions, it becomes important to reduce overall mission risk. As a result, we seek a design that is inherently robust, simple and low-mass to be a payload on a larger mission.

\section{The Tethered Axel ConcePt}

\section{Mission Concept}

Because of the steepness of the terrain we seek to access, we designed our rover to be tethered to a host platform. By only requiring a single fixed anchor point that can support the mass of Axel, the host platform can be a lander, a larger rover, or a habitat. Figure 5 shows a rendition (not to scale) of an overall mission concept where Axel is a payload on a Mars Science Laboratory-sized rover [9]. Separating the sample retrieval rover from the carrier rover improves overall mission safety by confining the risk of exploring steep terrain in the smaller rover.

In this mission scenario, a larger rover with a total payload of $65 \mathrm{~kg}$, such as that of the Mars Science Laboratory rover, may carry a $10 \mathrm{~kg}$ Axel rover for collecting samples from terrains that will be too risky for the larger rover to access. With only a fixed tether hook to the host rover, Axel can unreel its own tether over promontory or cliff walls, traverse over the rocky crater terrain, drive into the soft crater floor, collect soil samples, and reel itself back to the host rover.

There are fewer failure modes with Axel compared to a traditional rover. Fortunately, some of these failure modes are recoverable. First, the rover may sink into an area with soft sand. The Mars Exploration Rover (Opportunity) was trapped in a soft dune for several weeks in the spring of 2005. In such situations, Axel would use its tether to pull itself out even when there is no traction on its wheels. Second, the rover may encounter an obstacle that is too large to traverse. Similar to traditional rovers, Axel would use its on-board stereo vision and navigation software to detect and avoid such obstacles. Third, the tether may get entangled. Axel would use its link to keep the tether in tension. With its full range of motion, Axel can use its mobility to try to untangle the tether. Fourth, the tether may break. Axel would still be able to operate without a tether. However, its vertical mobility will be limited and it may not be able to return to the host platform on some steep terrains. Fifth, one of its three actuators may fail. Failure of a single drive wheel can be compensated for to some degree by the link actuator, but a failure of the link actuator will lead to an end to the tethered operations. 


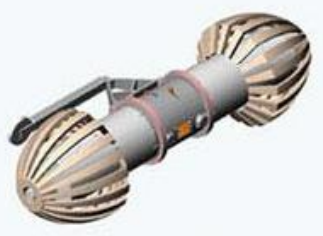

Axel Folded

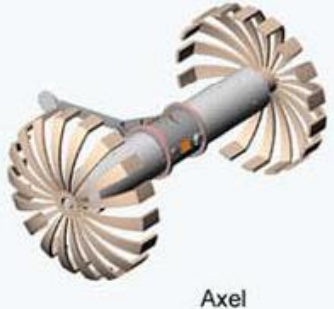

Axel
Figure 6-The Axel rover folded in its launch configuration (left), unfolded for surface deployment (right)

For an even higher level of reliability, one can conceive of missions with multiple Axels to provide system-level redundancy in case of a single Axel failure.

\section{Axel Description}

A primary goal of the Axel system design is minimal complexity and mass. Axel is fundamentally a two-wheeled rover with a symmetrical body and a trailing link. It uses three actuators to control its wheels, trailing link, and tether. The link serves several purposes: it reels and unreels the tether, it provides a reaction lever arm against wheel thrust on flat terrain, it adjusts the rover's pitch for pointing its stereo cameras and other instruments, and it provides some redundancy if one of the wheel actuators fails (see driving modes in the next section). Using only three actuators, this rover is capable of following arbitrary paths, turning-inplace, and operating upside-down or right side-up. Axel turns using differential driving of its wheels. This eliminates the need for complex steering mechanisms, which reduces complexity, mass and power consumption.

Based on the experience with Dante II, placing the tether on the rover would be significantly more reliable despite the additional mass of the cable and winch system. It is the alternative of reeling from the top that will subject the cable to severe degradation through the constant shearing of the cable against the sharp edges on the crater's rim, thus risking cable breakage.

Axel can readily support different wheel types and sizes ranging from large foldable wheels to inflatable ones. Hence, we can match wheel type and size to our best knowledge of the terrain characteristics. By using the drive motors on the wheels together with a locking mechanism, we envision the possibility of dynamically adjusting the wheel sizes from the small diameter wheels of the stowed configuration to the large diameter wheels of the unstowed configuration. However, this is currently only at the conceptual level. Figure 6 (left) shows Axel folded in a flight-packaging configuration. Figure 6 (right) shows Axel in its deployed configuration with its large low-inertia wheels ready for surface driving.

Because the Axel wheels envelope the rover's cylindrical body, depending on the compliance of the wheels, Axel can tolerate strong impacts during driving or landing. The colocation of its sensors, actuators, electronics, power, and payload inside the central cylinder simplifies the thermal control of the avionics, which is critical for planetary environments that expose the rover electronics to temperature extremes. In the Axel design, there is no need for wires to go outside the central cylinder thus eliminating the heat loss that would otherwise occur.

The Axel rover is equipped with computational and communication modules, stereo cameras, and an inertial sen sor for autonomous navigation with obstacle avoidance.

\section{Driving Modes}

The alignment of the rotational axes of the three actuators provides Axel with two driving modes. It also provides Axel with redundancy for its drive wheel actuators, where the link actuator can compensate for a failed drive actuator. Free of any tether (or with a very slack tether), Axel has two primary driving modes: rolling and tumbling.

In rolling mode, the wheel motors are actuated to drive the rover. With no tension on the tether, the trailing link rests on the ground. The wheel motor rotation, coupled with the reaction from the ground on the link, moves Axel in the forward direction. In this mode, Axel's cylindrical body will maintain a constant pitch throughout the motion.

In tumbling mode, only the link motor is actuated, which will then push the link into the ground. The result is a forward motion of the rover. In this mode, the wheels do not move relative to the rover body. However, the entire fixed wheel-body system rotates around the link. This tumbling mode is used to reel and unreel the tether on the rover's cylindrical body. In this mode, Axel has limited maneuverability as it can only move in straight lines forward and backwards.

When driving on slopes, both rolling and tumbling modes of driving, are used. Switching between these two modes is governed by terrain resistance and tether tension. On vertical walls where there is no terrain resistance or wheel traction, tumbling is the only mode available for mobility.

In rolling mode, Axel is capable of following arbitrary paths: driving straight lines, driving along arcs, turning-inplace, and following continuous trajectories. It can also drive in both directions. When Axel changes driving direction on flat terrain, the lightweight link is first flipped over from one side to the other. Once the link makes contact and provides a reaction force, Axel starts moving in the opposite direction.

To adjust the body pitch of the rover, we drive both the link actuator and wheel drive actuators in opposite directions. The result is a change in the rover's pitch without generating any forward or backward rover motion. Because 


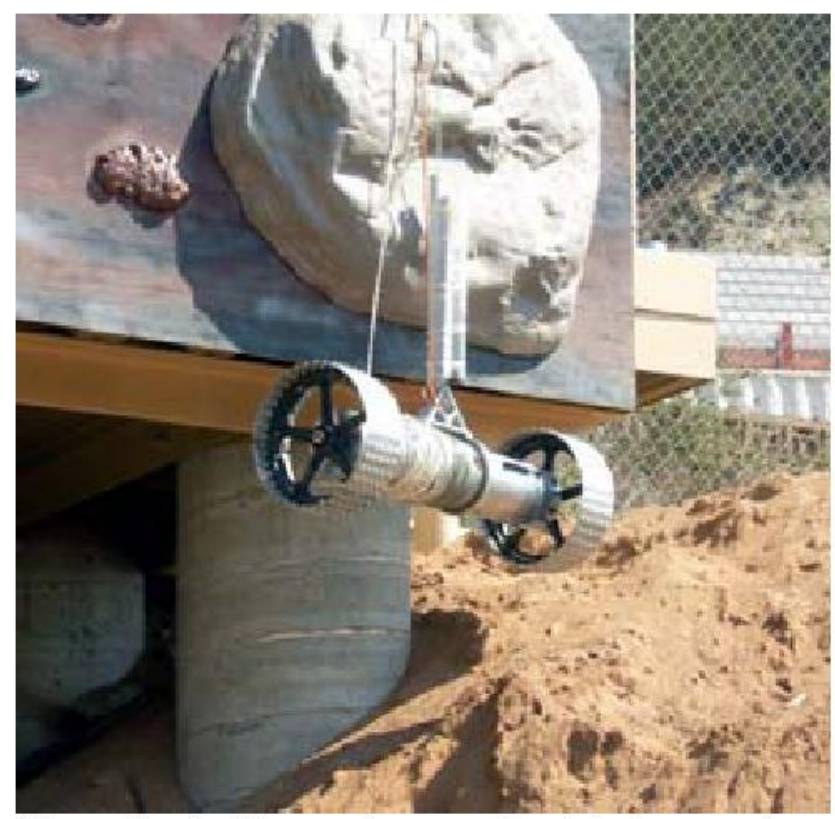

Figure 7-Axel lowered over a simulated promontory mounted on the viewing platform in the JPL Mars Yard.

Axel is symmetrical and has the ability to rotate its body a full $360^{\circ}$, there is no upside down or right side up state except as it relates to the direction in which the tether is reeled. By using hemispherical wheels on either end, Axel could operate in any configuration and hence support sudden drops.

\section{Tethered Operations}

Fundamentally, the tethered Axel operates like a yoyo. Axel uses its cylindrical body to wind the tether. The tether passes through the hollow link. Moving the link relative to the rover's body reels and unreels the tether.

On slopes, Axel uses a hybrid tumbling and rolling mode to simultaneously unreel its tether and drive down the steep terrain. Because Axel would roll on steep slopes, the tether tension will counterbalance the rolling motion keeping the link off the ground. This prevents the tether for getting tangled.

There are two configurations for reeling the tether: clockwise and counterclockwise. It is necessary for Axel to be able to operate with both winding configurations because when Axel transitions from free hanging to sloped terrain, one cannot control which side the rover will face when it enc ounters the surface of repose. Figure 7 shows Axel in a free hanging state after descending from an overhang simulating a crater promontory. Because the rover can freely twist on the tether, the rover can end up face up or down relative to the tether winding. However, the preferred configuration in the absence of free hanging stretches would be to unreel the tether in the same direction as the slope tumbling direction. This configuration has several

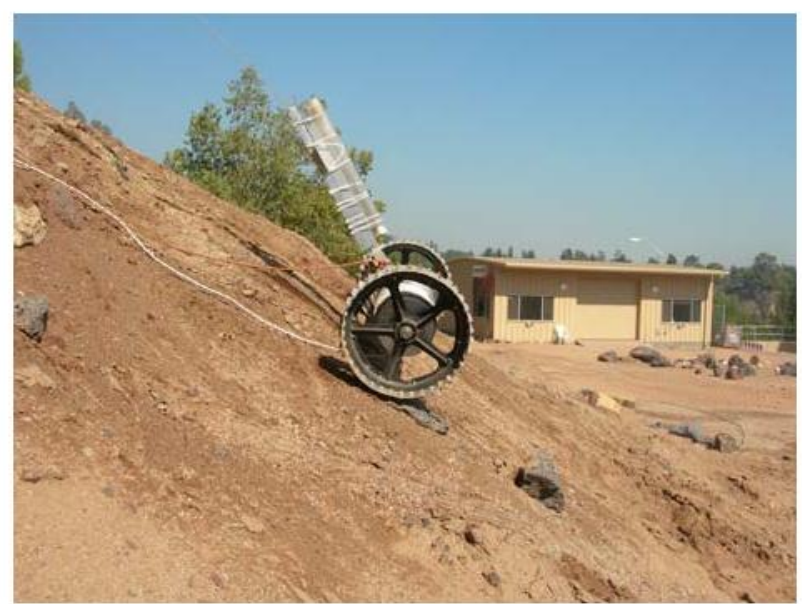

Figure 8-The Axel rover prototype driving down a $40^{\circ}$ slope.

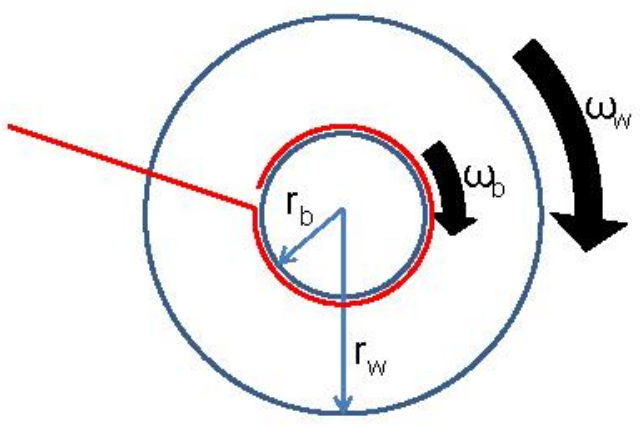

Figure 9-A cross section of the Axel body showing the wheel diameter, the cylindrical body diameter and the tether winding around the body

advantages for rolling down on slopes and for rolling off rocks if the rover high centers.

To properly control Axel's slope driving, the rover body needs to turn at a higher rate than the wheels relative to the ground. The ratio of the link to wheel motions is governed by the body and wheel diameters, and is necessary to maintain proper terrain traction without slippage. The mathematical formulation is presented in the next section.

\section{Theoretical Analysis}

A theoretical analysis of Axel's kinematics and dynamics can help build a better understanding and intuition of the rotation angles, intrinsic forces, and constraints that define this particular rover's motion. For this reason, a brief overview of the simplified physics model governing this robotic system is offered.

Figure 8 shows the tethered Axel rover prototype driving down an approximately $40^{\circ}$ slope. Our existing prototype currently has wheels with a diameter that is $50 \%$ smaller than what we desire. The steel tether runs through the hollow link coming out of the top end and is barely visible 


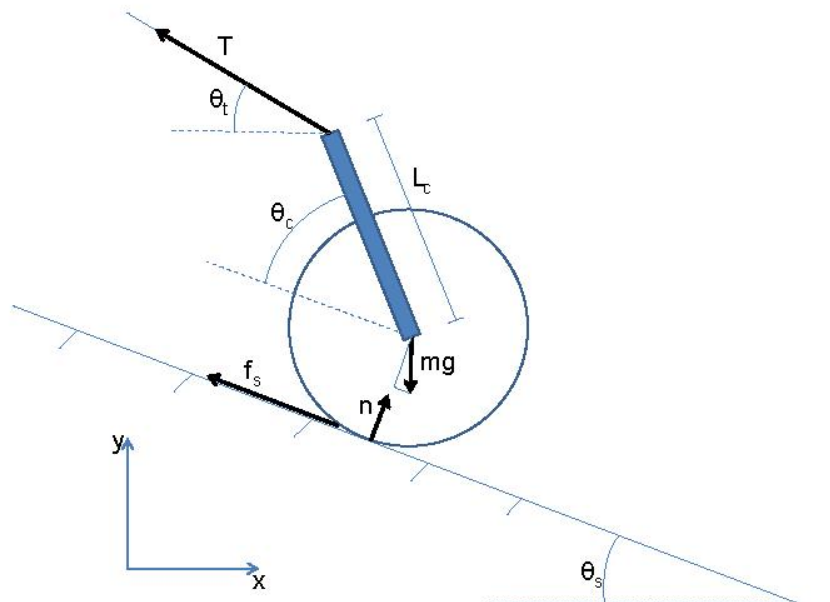

Figure 10-Free body diagram of the forces on Axel driving down a slope

in the picture. The other two slack cables are temporary and are not relevant to the control of the rover. The orange cable is our safety tether used in testing to prevent hardware damage or human injury if the steel tether fails. The white cable is a temporary power cable, which will be phased out once our on-board battery operated power system is completed.

Figure 9 represents Axel viewed along its axis of symmetry without the link. The subscript $b$ refers to properties of the body while the subscript $w$ refers to properties of the wheel. During tethered operation, the distance traveled by the rover is equal to the amount of tether that is reeled in or out. Analogously, Axel's linear wheel velocity is equal to the body's linear velocity, but their angular velocities have to be different because the tether is wound around the smaller body diameter. As a result, an important factor in controlling Axel is determining the speed at which to actuate the wheel motors such that the wheels maintain nonslipping ground contact at all time. If we assume that the tether is under tension, the body rotation will be equal to the actuated link rotation. Thus, for a given body rotation, $\omega_{b}$, we would like to calculate the wheel actuation, $\omega_{\text {act }}$, that prevents Axel from slipping. For motion without slipping, we have:

$$
\begin{gathered}
v_{b}=v_{w} \\
\omega_{b} r_{b}=\omega_{w} r_{w} \\
\omega_{b}=\frac{r_{w}}{r_{b}} \omega_{w}
\end{gathered}
$$

Computing $\omega_{w}$ we find that:

$$
\omega_{w}=\omega_{b}+\omega_{a c t}
$$

Substituting equation 2 into equation 1 and solving for $\omega_{\text {act }}$ will yield the following result:

$$
\omega_{a c t}=\omega_{b}\left(\frac{r_{b}-r_{w}}{r_{w}}\right)
$$

This proportionality is easily implemented in the control software. It is worth noting that since we are assuming the tether is under tension, Axel will travel at speed $v_{b}$ regardless of the wheel rotation speed. This assumption is valid for conditions where the gravity force dominates over the friction force on the wheels, i.e. steep slopes over loosely packed sand. Although the wheels are spinning, the force for motion is derived entirely from gravity (descent) and the tension in the tether (ascent).

Figure 10 is a basic free body diagram for a tethered Axel on a slope of angle $\theta_{\mathrm{s}}$. $\theta_{t}$ is the angle between the tether and the horizontal and is not necessarily equal to $\theta_{\mathrm{s}}$. We will assume that the mass of the body is much greater than that of the link so that the center of mass of the system is not shifted off the axis of rotation. Traveling at constant velocity, the sum of the forces and moments about the center of mass will be zero:

$$
\begin{aligned}
\sum F_{x}=0 & =-T \cos \theta_{t}-f_{s} \cos \theta_{s}+n \sin \theta_{s} \\
\sum F_{y}=0 & =T \sin \theta_{t}+f_{s} \sin \theta_{s}-m g+n \cos \theta_{s} \\
\sum M=0= & T \cos \theta_{t} L_{c} \sin \left(\theta_{c}+\theta_{s}\right) \\
& -T \sin \theta_{t} L_{c} \cos \left(\theta_{c}+\theta_{s}\right)-f_{s} r_{w}
\end{aligned}
$$

Since we consider the mass, angles, and lengths known quantities, we have three equations with three unknown forces. After some manipulation, we can express these forces in terms of the known quantities:

$$
\begin{aligned}
& n=\frac{m g\left(r_{w} \cos \theta_{t}+L_{c} \cos \theta_{s} \sin \left(\theta_{c}+\theta_{s}-\theta_{t}\right)\right)}{L_{c} \sin \left(\theta_{c}+\theta_{s}-\theta_{t}\right)+r_{w} \cos \left(\theta_{s}-\theta_{t}\right)} \\
& T=\frac{m g r_{w} \sin \theta_{s}}{L_{c} \sin \left(\theta_{c}+\theta_{s}-\theta_{t}\right)+r_{w} \cos \left(\theta_{s}-\theta_{t}\right)} \\
& f_{s}=\frac{m g L_{c} \sin \theta_{s} \sin \left(\theta_{c}+\theta_{s}-\theta_{t}\right)}{L_{c} \sin \left(\theta_{c}+\theta_{s}-\theta_{t}\right)+r_{w} \cos \left(\theta_{s}-\theta_{t}\right)}
\end{aligned}
$$

\section{The Axel Platform Prototype}

We transformed the original Axel modular rover to a tethered rover for sampling on steep terrain. We use the center of the Axel cylindrical body as a winch with the actuated link to reel and unreel the tether. The tether runs through the hollow link. We placed a sampling device alongside the link. The device is a simple passive cylinder with an inset funnel at the far end of the link. The funnel traps soil samples in the caching cylinder. With this device, we are able to collect samples from multiple sites. Currently, our sampling device does not separate samples from the multiple sites, but we plan to do so in the future.

The central cylinder of Axel is an aluminum tube with an outer diameter of 6 inches $(15.24 \mathrm{~cm})$, thickness of 0.125 inches $(0.31750 \mathrm{~cm})$, and a length of 2 feet 8 inches $(81.28$ $\mathrm{cm})$. The wheels are approximately $32.7 \mathrm{~cm}$ in diameter with a width of $13.4 \mathrm{~cm}$. The link used in these experiments extends $69.6 \mathrm{~cm}$ from the axis of the cylinder. When the 
support tether is used, it passes through the length of the link. This allows the rotation of the link to wrap or unwrap the tether around the main body. The tether used is a $1 / 8$ inch clear-vinyl coated $7 \times 7$ stranded steel fiber cable rated for 920 pounds. The $35 \mathrm{~m}$ section of the tether that was used in the Axel experiment weighed a total of $1 \mathrm{~kg}$.

Two 30V 153W servodisc drive motors from Kollmorgen drive the Axel's wheels. One 24V 150W Maxon motor powers the link. All three motors have 500 count encoders. These motors and encoders are controlled and monitored by two ESC629 PC/104 motor controllers from Real Time Devices. Axel's CPU board is a $700 \mathrm{MHz}$ Pentium processor from Advanced Digital Logic with $128 \mathrm{MB}$ of RAM with 2 GB flash drive. The software is implemented as combination of $\mathrm{C} / \mathrm{C}+\mathrm{H}$ running on the Debian Linux operating system.

Communication with the rover is handled through wireless Ethernet using a LinkSys $802.11 \mathrm{~b}$ bridge. Although not used in these experiments, the rover is also equipped with two black and white Dragonfly Firewire cameras from PointGrey with a resolution of $640 \times 480$ pixels. The cameras are connected to a MSMW104+ FPC-104 Firewire card.

Axel is currently powered through an external power supply with a non-weight bearing cable connected to the Axel body. A Parvus (PRV-0975X-01) DC/DC power converter converts the $24 \mathrm{~V}$ input to the internal voltages that are needed to operate Axel's avionics.

\section{Experimental Results}

We have conducted a series of experiments with the Axel rover. The very first set of experiments was conducted between $2002-2004$ on flat terrain in an indoor laboratory environment and later on in the JPL phase II Mars Yard. Initial tests were conducted with the un-tethered Axel. Both indoor and outdoor experiments showed that Axel is capable of driving over rocks that are about a $1 / 3$ wheel diameter. Axel drove over $20 \mathrm{~m}$ of rock-strewn terrain in the phase II JPL Mars Yard. Axel successfully traversed the terrain using tele-operation of the vehicle. For rock exceeding $1 / 3$ wheel diameter, it was necessary to control the link in order to avoid rolling it over the top and through a $225^{\circ}$ angle. Even though the roll does not harm the rover, we wanted to avoid this interval of uncontrollable state. Therefore, controlling the link played a key role in these experiments. Then, it is not surprising that controlling this link plays an even more critical role in the tethered Axel experiments.

The second set of experiments was conducted with the tethered rover over the summer of 2007. Axel was tested extensively in both indoor and outdoor environments and preliminary data was collected in order to quantify the rover's performance.
Twelve runs were conducted to measure Axel's power consumption and efficiency: three for each type of driving mode (rolling or tumbling) on both flat and sloped terrain. For all of the tests, Axel was running at 24 Volts. Maximum current measurements were recorded under each specific operating condition. Thus, the data represents an upper bound for the power usage and worst-case scenario in terms of efficiency. The figures are also normalized in terms of distance and weight in order to provide a basis for comparison to other rovers. The data from tests conducted on loose sand and packed dirt with $0^{\circ}$ and $13^{\circ}$ of inclination, respectively, is enumerated in Table 1.

\section{Table 1-Comparing Energy Costs}

\begin{tabular}{|c|c|c|c|c|}
\hline Rover & $\begin{array}{l}\text { Drive } \\
\text { Mode }\end{array}$ & Ground Type & $\begin{array}{c}\text { Ground } \\
\text { Inclination }\end{array}$ & $\begin{array}{l}\text { Ave. Max Energy Cost } \pm \\
\text { Standard Error } \\
\left(\mathrm{J} /\left(\mathrm{kg}^{\star} \mathrm{m}\right)\right]\end{array}$ \\
\hline Axel & Rolling & \begin{tabular}{|l|} 
Loose sand \\
\end{tabular} & $0^{\circ}$ & $35.55 \pm 1.05$ \\
\hline Axel & Tumbling & Loose sand & $0^{\circ}$ & $19.71 \pm 0.46$ \\
\hline Axel & Driving & Packed Dirt & $13^{\circ}$ & $35.84 \pm 0.5$ \\
\hline Axel & Rolling & Packed Dirt & $13^{\circ}$ & $28.42 \pm 2.03$ \\
\hline Rover & $\begin{array}{l}\text { Drive } \\
\text { Mode }\end{array}$ & Ground Type & Inclination (D) & $\begin{array}{l}\text { Average Energy Cost } \\
\left(\mathrm{J} /\left(\mathrm{kg}^{*} \mathrm{~m}\right)\right]\end{array}$ \\
\hline Rocky 8 & Rolling & Loose sand & $0^{\circ}$ & 21.38 \\
\hline
\end{tabular}

On both flat terrain and over a slight incline, driving Axel in tumbling mode used significantly less power and proved more efficient than the rolling drive mode. This is attributed to the fact that the link has a smaller actuator with a higher output torque but slower speed compared to the drive wheel actuators. The original Axel was designed to move large payloads relatively fast, which required higher speed and more powerful motors to drive the wheels. The calculated upper bound values for power and efficiency for the Axel platform are on par with those of Rocky 8 (see Table 1).

Axel's dynamics are shifted dramatically when the rover is tethered, and an upgrade to the control software was required in order to properly maneuver the rover. In tethered operation, a simultaneous combination of wheel and link rotation is required in order to travel without slipping on the ground. The advantage to this mode of operation is that more extreme terrain can be traversed with relative ease due to leverage from the tension in the tether.

Tethered operation was tested on various slopes with different ground types. We have conducted around fifteen runs on a $3 \mathrm{~m}$ relatively flat stretch of $15^{\circ}-20^{\circ}$ slope made up of packed dirt. Another eight runs were conducted on a 5 $\mathrm{m}$ slope varying in inclination from 0 to 40 degrees (Figure 1). These experiments took place in the JPL Mars Yard and covered terrain made up of loosely packed sand on undulated slope sprinkled with a number of medium sized rocks (1/3 wheel diameter). Excursions ranged from $10-25$ $\mathrm{m}$ round trip and included several maneuvers on the slope to 


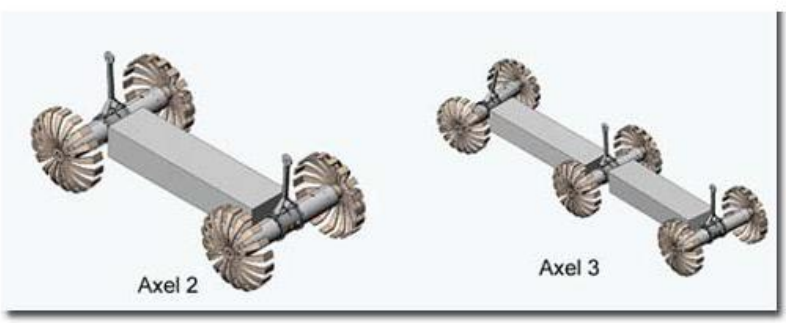

Figure 11-Two Axels docked to each side of a payload module forming an Axel2 rover (left), an Axel3 rover with two payload modules and three Axels

verify Axel's capabilities. These included manually avoiding hazardous rocks, turning in place while on a tether, arc driving, sampling, driving in both directions, and sampling. These maneuvers where carried out on both sloped and flat terrain with a taut and slack tether respectively.

The tests on all slopes proved very successful. Axel managed to both ascend and descend the steep terrain comfortably, even over rocks that were approximately $1 / 3$ of the wheel diameter.

Axel was also tested on a $90^{\circ}$ slope (freely hanging from the tether) in the lab and in the field as shown in Figure 7. By reeling and unreeling the tether through link actuation, the rover was able to raise and lower itself in a stable and controlled fashion without requiring any surface contact.

In addition to traversing steep and rocky terrain, Axel has the ability to sample dirt at any location along its path. Each scooping operation would collect a sample of about $30-40$ $\mathrm{g}$ of soil. Testing of Axel's sampling capability was conducted on all slopes during approximately half of the experiments. Both single samples and multiple samples were collected at various incline points in a single run but the samples were stored in the same sample-caching cylinder. Because our sampling device was targeted for sloped terrain, collecting samples on flat terrain with such a device proved difficult. The sampling device was designed to push the sampling cylinder into the ground of the sloped terrain. Doing so on the flat terrain requires simultaneously driving of the link into the ground and moving Axel in the same direction. Because the weight of the rover was not being supported by the link, it tended to lift off the ground and thus failed to obtain a sample. Sloped terrain was much more conducive to collecting soil samples. After pointing the link downhill and loosening the tension in the tether, raising the arm would drive the sampling device into the soil. Collecting sediment in this manner was very successful on all slopes greater than $10^{\circ}$. Sampling at two different locations during the same run, was neither more nor less difficult than single stage if both testing locations met the minimum slope requirement.
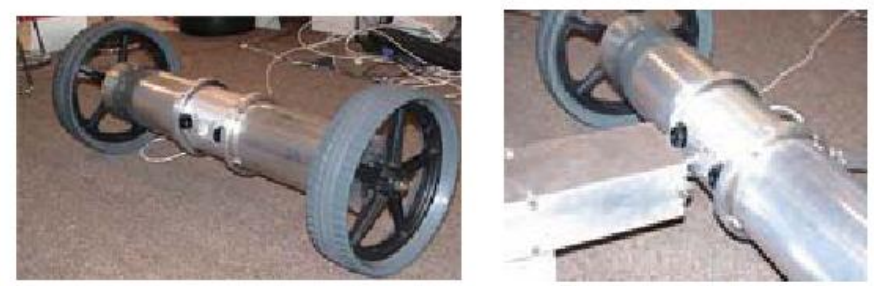

Figure 12-Original Axel (left), Axel docking to a payload module (right)

While sampling was successful on slopes that met the minimum grade requirements, sampling on flat terrain was not successful with the current sampling device. A second failure mode was the result of a systematic failure of the avionics that causes the on-board processor to reboot. This situation occurred a few times during the final ascent stages on a vertical wall with a large rock overhang. We believe that the un-chamfered link could have been caught on a rock crevice. Under such conditions, the CPU would sometimes reboot resulting in loss of control of the rover and engaging of the safety tether. We hypothesize that an overhang impediment resulted in the motors drawing excessive current, which in turn caused a decrease in current flow to the CPU. Further investigation would be required to determine the true nature of this failure and find a solution.

\section{FUTURE WORK}

While the initial set of experiments was successful in demonstrating Axel's mobility on slopes ranging from $0^{\circ}-$ $90^{\circ}$ and sampling on slopes ranging from $10^{\circ}-40^{\circ}$, experiments with longer traverses sampling on steeper slopes are desirable. This would ensure better terrain coverage for the current design. We would also like to further investigate science driven sampling requirements of potential future missions. A second critical step would be to demonstrate such experiments under autonomous control compared to the tele-operation control that we used during these experiments.

In the short term, we also plan to upgrade the current Axel prototype. First, we plan to use an on-board battery operated power system thus eliminating the need for an external power cable. Second, we plan to use more power efficient but slower drive wheel actuators. We also plan to relocate the cameras to avoid their being blocked by the tether. Third, we plan to design and deploy larger foldable wheels and carry out deployment experiments.

We also plan to explore new applications for Axel. Given its vertical mobility without the need of a surface to rest on, Axel is suitable for cave exploration on Mars and other planets. It has potential for urban search and rescue applications on Earth. 
Another major advantage of the single Axel design is its potential use as a part of a larger system. Axel rovers canbe arranged in a farrily of configurations to carry larger payload modules on the Lunar surface, as shown in Figure 11 and Figure 12 [3]. With either manual or autonomous in field docking, Axels can be used for a variety of exploration purposes and $s$ ientific investigations.

The mobility of the extended Axel rovers tums out to be similar compared to those irvestigated by General Motors decades ago [10].

\section{SUMMARY}

The discovery of bright new deposits in two gullies on Mars generated irterest to access and sample such material and motivated the development of a low-mass rover that can be hosted on a future mission to Mars. We have developed the Axel rover with a simple yet robust design to be able to traverse challenging terrain irside crater walls, collect samples and retum them to the mother ship. We have modified and tested the Axel rover prototype for tethend operations on steep te rain. We have conducted over two dozen experiments in the JPL Mas Yard diving the rover over the steepest slopes, collecting samples and asending back over a simulated promontory. We have de monstrated Axel's ability to descend $90^{\circ}$ hard-packed terrain, trarsition to very soft sand on steep terrain, collect samples and ascend back over a simulated promontory. Samples of about $40 \mathrm{~g}$ of soil where collected All experiments were carried out using tele-operation of the Axel rover.

\section{ACKNOWLEDGMENT S}

We would like to thank the team who was resporsible for the design, analysis and implementation of the original Axel rover: Dan Helmick, Prof. Ayama Howarl (formerly at JPL) of Georgia Tech, Prof. Raymond Cipra at Purche Unive sity, Prof. Murray Clark at Arkansas Tech Uriversity and Ed Barlow. We would also like to thank students: Raymond Christian, Damon Sisk, Isaac Vaughr, Samuel Irwin, Chad Keser, and Lauren Lyors.

Additionally, we would also like to ackrowledge the support of Prof. Chris Adari of Caltech and Dr. Neville Marzwell, Dr. James Cutts, Dr. Samad Hayati, Dr. Fichard Volpe and Dr. Gabriel Udomkesmalee, of JPL for their support of this work and for providing the JPL rower-testing infrastructure. We would like to thank Dr. Volpe who was the first to propose the ide a of nuning the te ther through the link.

This work is a joint collaboration be tween Caltech and the Jet Propulsion Laboratory. The work was dore at the Jet Propulsion Laboratory, Califomia Institute of Technology, under contract to the National Aerorautics and Space Adrinistration.

\section{REFERENCES}

[1] J. Bares, D. Wettergreen,"Dante II: Techurical Description, Results and Lesons Leamed," Intemational Joumal of Robotics Research, Vol. 18, No. 7, pp. 621-649, July, 1999.

[2] Stoe ter, S, and Papanikolopoulos, N.P., "Kinematic Mbtion Mbdel for Jurnping Scout Robots", IEEE Transactions on Robotics and Automation. Volume 22, No. 2, April 2006, pp 398-403.

[3] I. Nesnas, "Reconfigurable Exploratory Robotic Vehicles," NASA Tech Briefs, Jul 2001.

\section{[4] http:l/www.probotics.com}

[5] M Badescu, X. Bao, Y. Bar-Coher, Z. Chang, B. E. Dabiri, B. Kenredy, S. Sherrit, "Adapting the ultrasoniclsonic drillevicorer for walkingiclirbirg robotic applications," Proce edings of the SPIE, Volume 5762, pp. 160-168, 2005.

[6] P. Firjarian, C. Leger, E. Mum, B. Kerruedy, M Garrett, H. Aghazanian, S. Faritor, P. Schenker, "Distributed control for a modular, reconfigurable cliff robot", Robotics and Automation, 2002. Proceedings. ICRA'02. IEEE Intemational Conference on, Vol. 4 pp. 4083-4088, 2002.

[7] A. Howard, I.A. Nesnas, B. Werger, D. Helmick, "A Reconfigurable Robotic Exploration Vehicle for Extreme Environments," 10th Intemational Symposium on Robotics and Applic ations, Seville, Spain, June 2004.

[8] D. Bickler, "A family of planetary vehicles," Proc. of the Intem. Symp. on Mission Technologies and Design of Planetary Mbbile Vehic les, France, 1992.

[9] http:/imars.jpl nasa.gov/mslioverviewi

[10] http://www.lunarandplanetaryovers comiroverconcepts.ht m

\section{BIOGRAPHIES}

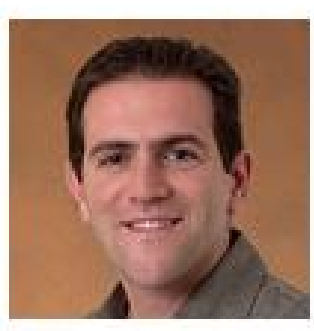

Issa AD. Nesnas, Ph.D is the principal invesigator for the Axel rover and a supervisor for the Robotics Software Systems Group. He has led robotics research progects at JPL for over eight years. Fis research interests include sensor-based robot control and software and hardware architectures for robotic systems. Issa received a 
B.E. degree in Electrical Engineering from Manhattan College, NY, in 1991. He earned the M.S. and Ph.D. degrees in Mechanical Engineering from the University of Notre Dame, IN, in 1993 and 1995 respectively. Prior to joining JPL in 1997, he worked as a senior engineer at Adept Technology Inc. Issa is a member of Eta Kappa Nu and Tau Beta Pi National Honor Societies.

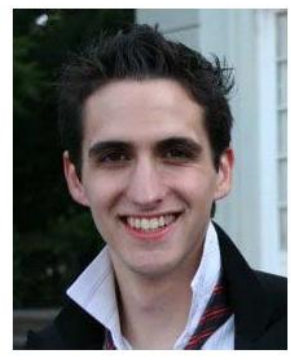

Pablo Abad-Manterola earned a B.S. in Mechanical Engineering and a minor in Mathematics from Stanford University in 2006 and moved to Pasadena to become a graduate student at the California Institute of Technology. He is pursuing an M.S. and Ph.D. in Mechanical Engineering under the guidance of Dr. Joel Burdick and with the support of a fellowship from the National Science Foundation. His research interests include autonomous sensor-based motion planning and multi-robot cooperation.

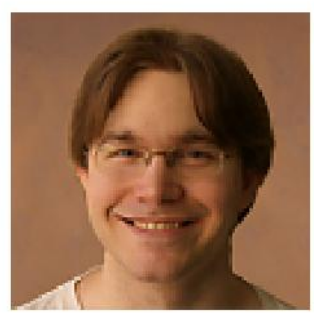

Jeffrey Edlund received his B.S. in Physics from Caltech in 2004. As a high school student he worked part-time building and maintaining Beowulf Supercomputing Clusters at the Center for Advanced Computing Research (CACR) at Caltech. Then, during his undergrad years he worked on data analysis algorithms for the Laser Interferometric Gravitational Observatory (LIGO). Upon completion of his undergrad work in 2003, he joined JPL section 3266 to continue working on gravitational wave science. He worked at JPL for 2 years before returning to Caltech to work with Dr. Adami and Dr. Burdick as a Graduate student in the Computation and Neural Science (CNS) program. Current research interests involve: biologically inspired developmental neural networks, digital evolution, and robotics.

Joel Burdick is Professor of Mechanical Engineering and BioEngineering at California Institute of Technology. 